\title{
Acta Cytologica
}

Aguilar-Jakthong, J.S. 348

Chen, M. 354

Chen, P. 310

Chin, C.D. 348

Chow, C. 348

Fang, F. 335

Feng, J. 310

Fu, X. 335, 354

$\mathrm{Hu}, \mathrm{J} .310$

Huang, Z. 310, 335

Kawashima, M. 342

Kezlarian, B. 324

Kiyuna, T. 342

Krane, J.F. 330
Lew, M. 286

Li, Q. 310

Li, Y. 348

Lin, O. 324

Liu, J. 335

Liu, N. 335

Lollie, T.K. 330

Lu, J. 310

Matsui, N. 342

McAlpine, E.D. 301

Michelow, P.M. 301

Ogura, M. 342

Osamura, R.Y. 342
Pantanowitz, L. 286, 301

Rao, E. 348

Rao, J. 283, 335, 348

Saiga, H. 342

Tang, G. 354

Wang, S.Y.(G.) 348

Wang, Z. 354

Wilbur, D.C. 286

Yu, W. 348

Zhang, Z. 335

\section{Subject Index Vol. 65, No. 4, 2021}

Algorithms 301

Artificial intelligence 286, 301, 310, 324, 342

Biomarkers 348

Bone marrow 354

Computational analysis 330

- cytology 286

Computer-assisted screening 286

Convolution neural network 310

Cytology 286
Deep learning 301, 310

Deep-learning algorithm 348

Digital cytology 301

- cytopathology 342

- imaging 335

- pathology 301,354

Fine-needle aspiration 330

Head and neck 330

Lymphocyte 354
Machine learning 324, 335, 354

Medical imaging 310

Molecular cytopathology 342

Morphological analysis 354

Multiplex immunofluorescence 348

Pap test 286

Salivary gland 330

Screening 286

Squamous cell carcinoma 330

Thyroid 330

- fine needle aspiration biopsy 324

Urine cytology 335

Urothelial carcinoma 335 